\title{
Populations of Xylella fastidiosa in Plants Required for Transmission by an Efficient Vector
}

\author{
B. L. Hill and A. H. Purcell
}

Department of Environmental Science, Policy and Management, University of California, Berkeley 94720-3112. Current address of B. L. Hill: Chiron Corporation, 4560 Horton St., Emeryville, CA 94608.

Accepted for publication 31 August 1997.

\begin{abstract}
Hill, B. L., and Purcell, A. H. 1997. Populations of Xylella fastidiosa in plants required for transmission by an efficient vector. Phytopathology 87:1197-1201.

Xylella fastidiosa, a xylem-limited bacterium that causes Pierce's disease (PD) of grapevine and other diseases, is transmitted efficiently by xylem-feeding leafhoppers. Acquisition of a PD strain of $X$. fastidiosa by the blue-green sharpshooter (BGSS) from five plant host species-grapevine (Vitis vinifera), Himalayan blackberry (Rubus discolor), California mugwort (Artemisia douglasiana), watergrass (Echinochloa crus-galli), and Bermuda grass (Cynodon dactylon) — was tested at various time intervals after vector inoculation. The minimum incubation periods in plant hosts before BGSS acquired $X$. fastidiosa were 4, 22, 29, and 25 days for
\end{abstract}

ABSTRACT

A xylem-limited bacterium, Xylella fastidiosa (26), causes Pierce's disease (PD) of grapevines, as well as diseases of alfalfa, almond, peach, citrus, forest trees, and other plants that are not economically important $(12,21)$. The bacterium is transmitted by xylem feeding, suctorial homopteran insects such as sharpshooter leafhoppers (family Cicadellidae, subfamily Cicadellinae) and spittle bugs (family Cercopidae) $(1,5,9,18)$. PD has spread to California vineyards from inoculum sources in plants outside the vineyard $(6,8,14)$, indicating that vector acquisition of $X$. fastidiosa from various plant species can be an important factor in the spread of crop diseases caused by this bacterium. In California, 20 species of xylem-feeding Cicadellidae and Cercopidae transmitted $X$. fastidiosa $(2,3,5)$. The principal and most efficient PD vector in coastal California is the blue-green sharpshooter (BGSS), Graphocephala atropunctata (Signoret) $(8,15)$. The most important PD vectors in other regions of California are the green sharpshooter (GSS), Draeculacephala minerva Ball, and the redheaded sharpshooter (RHSS), Carneocephala fulgida Nottingham $(7,8,20)$. Three studies have indicated that 94 of 151 plant species examined in California are potential hosts of $X$. fastidiosa $(4,23,24)$, but the epidemiological role and relative importance of these host plants in the spread of the bacterium to vineyards is poorly understood. $X$. fastidiosa multiplies and spreads faster and achieves higher numbers in some of these species (11). Plant species with higher bacterial populations and systemic infection are more likely to be important epidemiological sources of spread to grapevine only if higher bacerial populations lead to more efficient vector acquisition.

The objective of this study was to determine how the number of viable $X$. fastidiosa in host plants is related to vector transmission

Corresponding author : A. H. Purcell

E-mail address: purcell@ nature.berkeley.edu

Publication no. P-1997-1001-02R

(C) 1997 The American Phytopathological Society grapevine, blackberry, mugwort, and watergrass, respectively. There were no transmissions by vectors or recoveries of $X$. fastidiosa by culturing from Bermuda grass in 133 attempts, including 80 attempts with the green sharpshooter, Draeculacephala minerva. The first acquisitions and subsequent transmissions by BGSS occurred after $X$. fastidiosa multiplied to a population of about $10^{4} \mathrm{CFU} / \mathrm{g}$ of stem tissue. Higher populations of bacteria in plants resulted in higher rates of transmission. In grapevine, the rate of transmission increased over time $(4.5 \%$ in the first 10 days to $55 \%$ after day 25) as the maximum number of viable CFU of $X$. fastidiosa recovered by culturing also increased (from $5 \times 10^{5} \mathrm{CFU} / \mathrm{g}$ during the first 10 days to $5 \times 10^{8}$ after day 25 ).

Additional keywords: Carneocephala, Graphocephala, vector transmission. efficiency. Bacterial populations and efficiency of transmission of a PD strain of $X$. fastidiosa by BGSS and GSS were measured in five host plant species at various time intervals after insect inoculation of plants. Plant species tested included grapevine (Vitis vinifera L. 'Pinot noir'), Himalayan blackberry (Rubus discolor Weihe \& Nees), California mugwort (Artemisia douglasiana Besser), watergrass (Echinochloa crus-galli (L.) P. Beauv.), and Bermuda grass (Cynodon dactylon (L.) Pers.). These plants are common breeding hosts of the three most important California PD vectors (BGSS, GSS, and RHSS) $(7,16,20)$ and were used in a previous study $(11)$.

\section{MATERIALS AND METHODS}

Culture and enzyme-linked immunosorbent assay (ELISA). Numbers of viable $X$. fastidiosa (CFU per gram in plant samples) were estimated from the weight of the sample and the number of CFU per sample by dilution-plating homogenized samples on PWG culture medium as previously described (11). Colonies were counted after 7 days of incubation at $26^{\circ} \mathrm{C}$. The theoretical detection threshold for this method was calculated to be $\approx 100$ cultivable cells per $g$ of plant tissue or per sample (11). The presence of $X$. fastidiosa in plants also was verified by double-antibody sandwich ELISA. Methods for ELISA were as previously described (10). The ELISA detection threshold was about $10^{5}$ cells per $\mathrm{g}$ of plant tissue. For cases in which culture of plant samples yielded colonies but the ELISA results were negative, a second ELISA test was performed on the cultured cells to confirm the identity of the bacterium.

Vector, plant sources, and inoculation. In addition to the five plant species tested, alfalfa (Medicago sativa L. 'Moapa') was used in some experiments to verify the infectivity of vector insects, or it was inoculated and used as a source plant to infect vectors. The growth and maintenance of all plants and noninfective BGSS and GSS were as previously described (10). Infective BGSS were obtained by caging groups of up to 100 insects, 
which then fed for 2 to 6 days on each of two grapevines that exhibited PD symptoms and tested positive for X. fastidiosa by culturing and ELISA. Infective GSS were obtained by caging groups of GSS on infected alfalfa plants. Alfalfa did not exhibit noticeable disease symptoms (slow regrowth after cutting) until after 9 months, and plants used as acquisition sources tested positive for $X$. fastidiosa by culturing and ELISA. Vectors were verified as being inoculative as previously described (10). All acquisition plants were inoculated with a strain of $X$. fastidiosa we previously had isolated from a California vineyard and designated YVPD (11). Aliquots of a suspension of YVPD in phosphate buffer were stored at $-70^{\circ} \mathrm{C}$. The acquisition plants were inoculated either by needle (13), using a thawed aliquot of the stored cell suspension, or by insect vectors that had acquired the bacterium from needle-inoculated plants.

Experimental protocol. Experiments were replicated three to five times for each of the five plant species. Each replicate consisted of 12 to 30 experimental plants, depending on the number of inoculative insects available on a given date. Each experimental plant was inoculated by a group of four putatively inoculative insect vectors by caging the insects on a marked inoculation site of $2.5 \mathrm{~cm}$ of stem or petiole for an inoculation access feeding time of $48 \mathrm{~h}$. At various intervals after vector inoculation, groups of four noninfective sharpshooter leafhoppers (BGSS or GSS) were caged on the marked inoculation site and allowed to feed for 24 to $48 \mathrm{~h}$ to acquire $X$. fastidiosa. After these test insects were removed, some experimental plants (usually four or five) were collected, and their inoculation sites were tested by culturing and ELISA to determine the population of $X$. fastidiosa. The remaining experimental plants were held for later testing. After feeding on the experimental plant, the group of four test vectors was caged for 2 days on a susceptible indicator plant, either a grapevine seedling or a small (10 to $15 \mathrm{~cm}$ tall) alfalfa seedling. These indicator plants were tested 6 to 12 weeks later by culturing and ELISA for the presence of X. fastidiosa. We considered that transmission from the experimental plants had oc-

TABLE 1. On grapevine, number of transmissions and culture recoveries and maximum number of Xylella fastidiosa (CFU per gram) detected at 5-day postinoculation intervals

\begin{tabular}{lcccccc}
\hline & \multicolumn{5}{c}{ Time interval after inoculation of grapevine (days) } \\
\cline { 2 - 6 } & $1-5$ & $6-10$ & $11-15$ & $16-20$ & $21-25$ & $26-30$ \\
\hline Transmission attempts & 27 & 39 & 34 & 13 & 10 & $31-35$ \\
Transmissions & 2 & 1 & 1 & 4 & 1 & 10 \\
Culture attempts & 10 & 20 & 32 & 5 & $\ldots$ & 7 \\
Recoveries by culture & 1 & 7 & 27 & 5 & $\ldots$ & $\ldots$ \\
Maximum no. of CFU per gram & $2 \times 10^{4}$ & $5 \times 10^{5}$ & $1 \times 10^{7}$ & $1 \times 10^{8}$ & $\ldots$ & $5 \times 10^{8}$ \\
\hline
\end{tabular}

a Data not available.

TABLE 2. On blackberry, number of transmissions and culture recoveries and maximum number of Xylella fastidiosa (CFU per gram) detected at 5-day postinoculation intervals

\begin{tabular}{|c|c|c|c|c|c|c|c|c|c|}
\hline & \multicolumn{9}{|c|}{ Time interval after inoculation of blackberry (days) } \\
\hline & $1-5$ & $6-10$ & $11-15$ & $16-20$ & $21-25$ & $26-30$ & $31-35$ & $35-40$ & $>40$ \\
\hline Transmission attempts & 7 & 18 & 20 & 5 & 18 & 14 & 14 & 7 & 25 \\
\hline Transmissions & 0 & 0 & 0 & 0 & 1 & 4 & 0 & 5 & 8 \\
\hline Recoveries by culture & $\ldots$ & 0 & 0 & $\ldots$ & 0 & $\ldots$ & 3 & 3 & 19 \\
\hline Maximum no. of CFU per gram & & & & & & & $1 \times 10^{7}$ & $2 \times 10^{6}$ & $2 \times 10^{7}$ \\
\hline
\end{tabular}

a No data available.

TABLE 3. On mugwort, number of transmissions and culture recoveries and maximum number of Xylella fastidiosa (CFU per gram) detected at 5-day postinoculation intervals

\begin{tabular}{|c|c|c|c|c|c|c|c|c|}
\hline & \multicolumn{8}{|c|}{ Time intervals after inoculation of mugwort (days) } \\
\hline & $1-5$ & $6-10$ & $11-15$ & $16-20$ & $21-25$ & $26-30$ & $31-35$ & $>35$ \\
\hline Transmission attempts & $\ldots^{\mathrm{a}}$ & 7 & 30 & 5 & 24 & 12 & 26 & 25 \\
\hline Transmissions & 0 & 0 & 0 & 0 & 0 & 1 & 4 & 2 \\
\hline Recoveries by culture & $\ldots$ & $\ldots$ & 0 & $\ldots$ & 0 & $\ldots$ & 2 & 7 \\
\hline Maximum no. of CFU per gram & & & & & & & $2 \times 10^{5}$ & $3 \times 10^{5}$ \\
\hline
\end{tabular}

a No data available.

TABLE 4. On watergrass, number of transmissions and culture recoveries and maximum number of Xylella fastidiosa (CFU per gram) detected at 5-day postinoculation intervals, using blue-green sharpshooter and green sharpshooter insect vectors

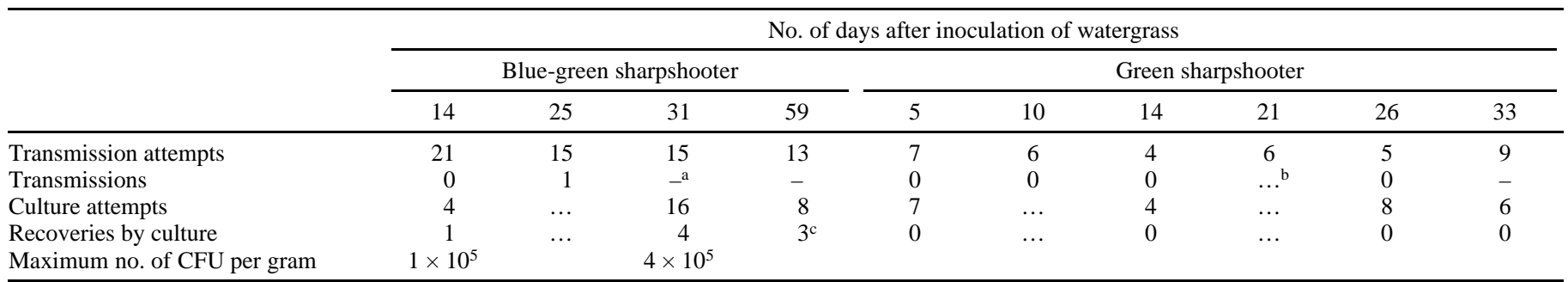

a Vector insects did not survive confinement on plants.

b No data available.

${ }^{c}$ These three recoveries were verified by enzyme-linked immunosorbent assay but not by culture, because the culture plates were overgrown with contaminants. 
curred when $X$. fastidiosa was recovered from the indicator plant.

All experiments on grapevine, blackberry, and mugwort were done with BGSS as the inoculative and test vector species. BGSS both feeds and breeds on these plants in nature $(15,16)$, and it reliably acquires $X$. fastidiosa and becomes infective when feeding on symptomatic grapevine or alfalfa $(10,19,25)$. We attempted to use GSS as the inoculative and test vector species in experiments with watergrass and Bermuda grass because this vector commonly occurs on these grasses in nature $(7,20)$. However, GSS did not become reliably inoculative when feeding on symptomatic infected grapevine. Two replicates each for watergrass and Bermuda grass, using GSS as the inoculative and test vector, were included, but for these experiments, the GSS were fed on infected alfalfa to become inoculative, and alfalfa was used as the indicator plant when testing for transmission. Three additional replicates each for watergrass and Bermuda grass used BGSS as the inoculative and test vector species.

\section{RESULTS}

The pooled totals of all replicates for each plant species for transmission attempts, transmissions, and culture attempts and recoveries and the maximum associated number of CFU per gram of plant tissue are summarized for grapevine, blackberry, and mugwort (Tables 1 through 3 ) in consecutive 5-day intervals after inoculation. Similar pooled results for watergrass (Table 4) are separated according to whether GSS or BGSS was used. The postinoculation incubation period in experimental plants before the first transmission (vector acquisition) of a PD strain of $X$. fastidios $a$ occurred and the incubation period before we first detected bacteria by culturing, respectively, was 4 and 5 days in grapevine, 22 and 32 days in blackberry, 29 and 31 days in mugwort, and 25 and 14 days in watergrass. No replicates differed significantly from the general trends observed over time for these four plant species. From Bermuda grass, there were no vector transmissions in 66 and 70 transmission attempts with BGSS and GSS, respectively, and there were no recoveries of $X$. fastidiosa by culturing or ELISA from Bermuda grass in 58 attempts. Transmission attempts after 30 days with BGSS from Bermuda grass and watergrass did not succeed because these vectors did not survive being caged for the 48-h acquisition feedings on the inoculation sites of these grasses. The inoculation sites by 30 days were senescent or dead in all

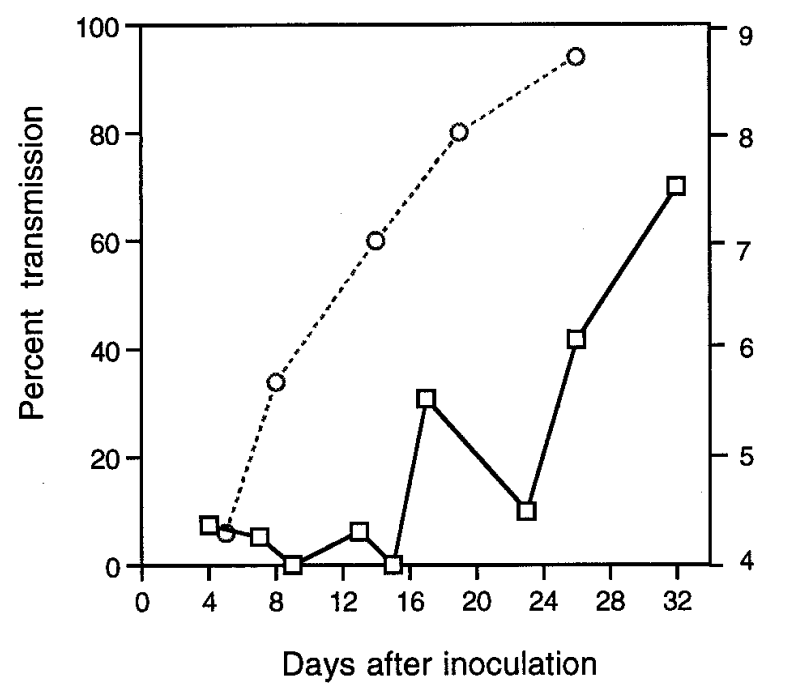

Fig. 1. On grapevine, populations (CFU per gram) of Xylella fastidiosa (dashed line) and vector transmission efficiency (solid line) after inoculation by infective blue-green sharpshooter insect vectors. trials. GSS survived confinement on these $>30$-day-old inoculation sites, but the trials did not result in transmissions. In grapevine (Fig. 1), the rate of transmission increased over time from about $5 \%$ during the first 10 days after inoculation to $55 \%$ after day 25 . The maximum number of viable $X$. fastidiosa recovered by culturing increased from $5 \times 10^{5}$ to $5 \times 10^{8} \mathrm{CFU} / \mathrm{g}$ during this period. During the first 10 days after vector inoculation of grapevine, BGSS transmission and recovery by culturing from these plants were both low (Fig. 2). Only when $X$. fastidiosa was recovered readily by culturing ( $100 \%$ of all attempts) was transmission efficiency greater than $25 \%$.

\section{DISCUSSION}

A postinoculation interval or incubation period was observed before the first transmission of the YVPD strain of $X$. fastidiosa occurred from each of the four host plant species infected. The incubation period before first transmission by vectors was similar in duration to the postinoculation incubation interval before first detection of the pathogen by culturing for these plants. Differences in the incubation interval before detection by culturing among the plant species suggests differences in the multiplication rate of this strain of $X$. fastidiosa within plants (11). The incubation period required for transmission suggests that the bacterium must multiply in the host plant to threshold population levels before vectors can efficiently acquire $X$. fastidiosa. Threshold population levels of $X$. fastidiosa for vector acquisition were close to the population levels first detected by culturing, $\approx 10^{4} \mathrm{CFU} / \mathrm{g}$ in our experiments. The bacterium multiplied most rapidly in grapevine, and the associated incubation period before transmission also was much shorter for grapevine than for other plants. Grapevine and blackberry supported higher populations of $X$. fastidiosa (11), and both plants also had higher rates of transmission by vectors than did mugwort and watergrass, which had lower bacterial populations. It seems reasonable to expect that bacterial multiplication would be necessary in a plant before onset of vector acquisition. However, it has been shown that another gram-negative bacterium, BEV, is acquired by uninfected leafhoppers from plants even though BEV neither multiplies within nor spreads beyond its inoculation site in plants (22). BEV is an unclassified member of the gamma subdivision of Enterobacteraceae and is a parasite of the

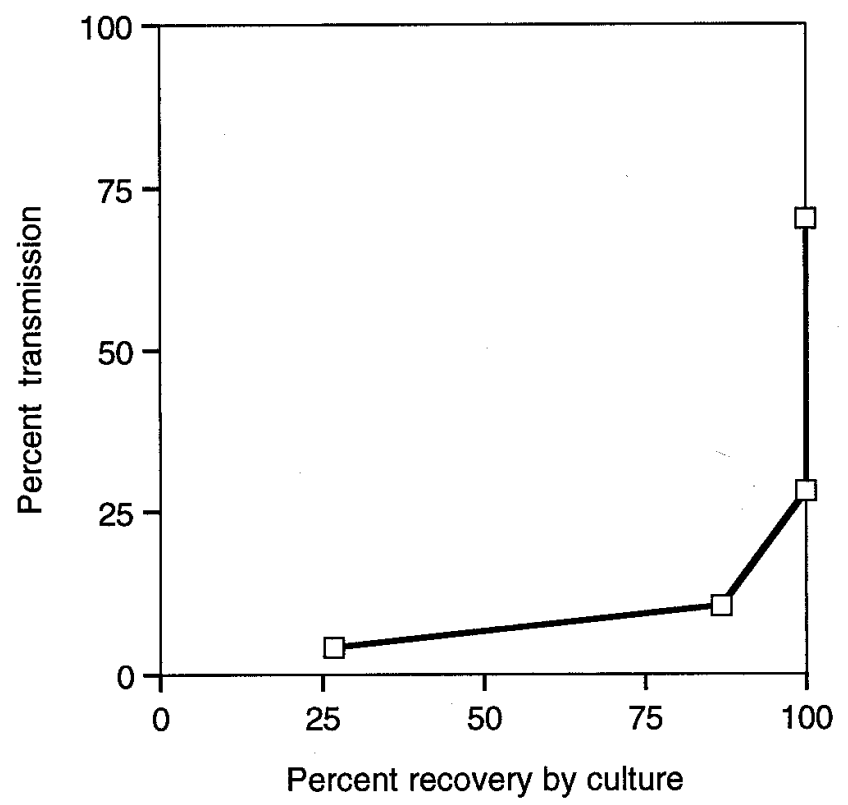

Fig. 2. On grapevine, transmission and recovery rate by culturing Xylella fastidiosa, grouped in 10-day postinoculation intervals. 
leafhopper Euscelidius variegatus. Uninfected leafhoppers acquire this bacterium from plants that have been inoculated by infected leafhoppers (22).

We did not detect $X$. fastidiosa in Bermuda grass by culturing, ELISA, or vector transmission, but Freitag (4) found it to be a host of the bacterium in his transmission experiments during the 1940s. Freitag's (4) experiments with Bermuda grass differed from our study in that groups of 5 to 20 infective GSS were caged on the whole plant and left (presumably for $\approx 2$ weeks) until they either oviposited or died. Nymphs emerging from the eggs of the inoculative GSS were removed after 2 to 4 weeks to a noninfected alfalfa plant. Subsequent symptom development in the alfalfa indicator plant was confirmation of transmission from Bermuda grass. The number of transmitting nymphs was not stated; however, based on our experience in rearing GSS on Bermuda grass, it is reasonable to expect that 50 to 300 nymphs might have been present. There are several possible explanations of why Freitag (4) found Bermuda grass to be a host and this study did not. (i) Transmission from Bermuda grass could be a low probability event, one that the greater number of insects and the longer inoculation and acquisition feeding times used by Freitag (4) would have been more likely to detect. (ii) The GSS in this study were confined to stem tissue near the crown of the plant, whereas in the Freitag study (4) the GSS were caged on the whole plant. It is possible that transmission from Bermuda grass is more frequent when acquisition is from leaves or other feeding sites. (iii) The Freitag (4) experiments were conducted during an epidemic outbreak during the 1940s and may have involved strains of $X$. fastidiosa that multiplied in Bermuda grass.

It was surprising that transmission rates from grapevine in this study did not approach $100 \%$. Groups of four uninfected BGSS were used to transmit $X$. fastidiosa from experimental plants. The decision to use groups of four vectors was made to increase the probability of transmission, because the transmission rate from nongrape plant species was expected to be low. The maximum rate of transmission (70\%) from grapevine occurred after 30-day incubation periods. Consequently, in $30 \%$ of the attempts none of the four BGSS individuals transmitted. In a group of four, when one or more of the individuals in the group can transmit and the transmission rate is $70 \%$ per group, the probability that any one individual in the group will transmit is $24 \%$ (calculated from the fourth-order binomial). Thus, the maximum transmission rate by individual vector insects from grapevine was $\approx 24 \%$, whereas in other studies the transmission rates for individual BGSS were 90 to $100 \%(10,19)$ with the same source plant populations of $X$. fastidiosa $\left(10^{8}\right.$ to $\left.>10^{9} \mathrm{CFU} / \mathrm{g}\right)$ and the same (or shorter) feeding times for acquisition and inoculation (24 to $48 \mathrm{~h}$ ).

One explanation for the lower transmission rate could be that efficiency of acquisition and inoculation may be related to the site on the plant where the vector feeds to acquire $X$. fastidiosa. In previous studies, vector insects were caged with access to the entire infected plant. We observed that BGSS fed on petioles, leaf veins, and the succulent stem near the growing top of the plant and avoided feeding on mature stem tissue closer to the soil. In contrast, we caged the test vector groups to acquire $X$. fastidiosa at the marked inoculation site on the stem, typically about half way $(7$ to $13 \mathrm{~cm}$ ) up a 15 - to $25-\mathrm{cm}$-tall grape seedling at the time of inoculation. The experimental plant also was growing during the 30 days postinoculation, so the stem sites at the time of the acquisition feeding for the transmission attempts were proportionately lower on the plant, typically $7-$ to $13-\mathrm{cm}$ up a $30-$ to $45-\mathrm{cm}$ tall grape seedling. Although it has not been experimentally tested, acquisition efficiency from these stem sites may be much lower than acquisition efficiency from near the growing top of the plant. If true, this may explain the low efficiency of acquisition of $X$. fastidiosa by GSS from grapevine.

The preferred hosts for GSS are grasses, and this vector's preferred feeding site is on stems near the crown of grass plants within 1 to $5 \mathrm{~cm}$ of the ground. When caged to feed on grapevine, they usually feed on stems near the ground. In these experiments, when BGSS and GSS were fed on infected grapevine, they were caged with access to whole plants. BGSS fed near the growing top of the plant, and GSS fed low, near the crown of the plant. The subsequently inoculative BGSS groups were confirmed to be almost $100 \%$ infective. In contrast, there were eight experiments in which the rate of infectivity of GSS groups was 0 to $5 \%$ (data not shown). GSS acquired X. fastidiosa efficiently only when fed on infected alfalfa (25; B. L. Hill and A. H. Purcell, unpublished data). We do not know if GSS would acquire $X$. fastidiosa more efficiently from grapevine if it were caged for acquisition near the growing top of infected grapevines.

In this study, (i) X. fastidiosa multiplied to threshold populations in plant hosts before transmission by vectors from these plants became likely. Transmissions did not occur before the populations of $X$. fastidiosa in test plants reached between $10^{4}$ and $10^{5} \mathrm{CFU} / \mathrm{g}$. (ii) The rate of transmissions increased as the population of $X$. fastidiosa in the plant increased. (iii) The rate of transmission from plants increased as the rate of recovery by culturing from the same plants increased. (iv) Insects fed on grapevine and blackberry, which supported larger populations of a PD strain of $X$. fastidiosa, also had higher rates of transmission than insects fed on mugwort and watergrass, which had lower bacterial populations. If the population levels and multiplication rate of $X$. fastidios $a$ within plants affect the rate of transmission by vectors, characteristic differences among plants in these traits should make some plant species, such as wild grapevine and blackberry, more important than other plant species as reservoirs for the spread of PD strains of $X$. fastidiosa to vineyards. In California many of the 94 plant species earlier identified as hosts of $X$. fastidiosa (reviewed in reference 11) may not support sufficiently high bacterial populations for significant acquisition by vector insects.

Bacterial populations fluctuate seasonally in wild plant hosts. For example, ELISA detected X. fastidiosa only from May through September from a natural population of blackberry plants that was sampled monthly, but detected the bacterium in periwinkle (Vinca minor) only during November through May (23). The latter finding was based solely on ELISA tests without negative periwinkle controls. This should be confirmed by culturing. Seasonal changes in populations of $X$. fastidiosa in plants are likely to be especially significant where vectors do not overwinter as adults, because critical early spring inoculations of grapevines (17) require plant sources of inoculum for potential vectors that become adults during winter or spring. Our results indicate that recovery of $X$. fastidiosa by culturing can be an effective predictor of the potential of various plants to serve as acquisition sources for insect vectors. This should facilitate future studies to establish which plant hosts are regular and important sources of inoculum for the spread of PD to vineyards.

\section{ACKNOWLEDGMENTS}

Research was supported in part by USDA Competitive Grant 90-371535435. Part of the Ph.D. dissertation research of B. L. Hill. We thank S. Saunders, M. Medeiros, A. Franco, and R. Martinez for their assistance. S. Lindow and T. Mittler made helpful suggestions.

\section{LITERATURE CITED}

1. DeLong, D. M., and Severin, H. H. P. 1950. Spittle-insect vectors of Pierce's disease virus. I. Characters, distribution, and food plants. Hilgardia 19:339-355.

2. Frazier, N. W. 1965. Xylem viruses and their insect vectors. Pages 91-99 in: Proc. Int. Conf. Virus Vectors Perennial Hosts Special Ref. Vitis. Division of Agricultural Science, University of California, Davis.

3. Frazier, N. W., and Freitag, J. H. 1946. Ten additional leafhopper vectors of the virus causing Pierce's disease of grapes. Phytopathology 36:634-637.

4. Freitag, J. H. 1951. Host range of Pierce's disease virus of grapes as determined by insect transmission. Phytopathology 41:920-934. 
5. Freitag, J. H., and Frazier, N. W. 1954. Natural infectivity of leafhopper vectors of Pierce's disease virus of grape in California. Phytopathology 44:7-11.

6. Goodwin, P., and Purcell, A. H. 1992. Pierce's disease. Pages 76-84 in: Grape Pest Management. 2nd ed. Division of Agriculture and Natural Resources, University of California, Oakland.

7. Hewitt, W. B., Frazier, N. W., and Freitag, J. H. 1949. Pierce's disease investigations. Hilgardia 19:207-264.

8. Hewitt, W. B., Frazier, N. W., Jacob, H. E., and Freitag, J. H. 1942. Pierce's disease of grapevines. Calif. Agric. Exp. Stn. Circ. 353:1-32.

9. Hewitt, W. B., Houston, B. R., Frazier, N. W., and Freitag, J. H. 1946. Leafhopper transmission of the virus causing Pierce's disease of grape and dwarf of alfalfa. Phytopathology 36:117-128.

10. Hill, B. L., and Purcell, A. H. 1995. Acquisition and retention of Xylella fastidiosa by an efficient vector, Graphocephala atropunctata. Phytopathology 85:209-212.

11. Hill, B. L., and Purcell, A. H. 1995. Multiplication and movement of Xylella fastidiosa within grapevine and four other plants. Phytopathology 85:1368-1372.

12. Hopkins, D. L. 1989. Xylella fastidiosa: A xylem-limited bacterial pathogen of plants. Annu. Rev. Phytopathol. 27:271-290.

13. Hopkins, D. L., Thompson, C. M., Bistline, F. W., and Russo, L. W. 1989. Relationship between xylem-limited bacteria and citrus blight. Proc. Fla. State Hortic. Soc. 102:21-23.

14. Purcell, A. H. 1974. Spatial patterns of Pierce's disease in the Napa Valley. Am. J. Enol. Vitic. 25:162-167.

15. Purcell, A. H. 1975. Role of the blue-green sharpshooter, Hordnia circellata, in the epidemiology of Pierce's disease of grapevines. Environ. Entomol. 4:745-752.

16. Purcell, A. H. 1976. Seasonal changes in host plant preference of the blue-green sharpshooter Hordnia circellata (Homoptera: Cicadellidae).
Pan-Pac. Entomol. 52:33-37.

17. Purcell, A. H. 1981. Vector preference and inoculation efficiency as components of varietal resistance to Pierce's disease in European grapes. Phytopathology 71:429-435.

18. Purcell, A. H. 1990. Homopteran transmission of xylem-inhabiting bacteria. Pages 243-266 in: Advances in Disease Vector Research, vol. 6. Springer-Verlag, New York.

19. Purcell, A. H., and Finlay, A. H. 1979. Evidence for noncirculative transmission of Pierce's disease bacterium by sharpshooter leafhoppers. Phytopathology 69:393-395.

20. Purcell, A. H., and Frazier, N. W. 1985. Habitats and dispersal of the leafhopper vectors of Pierce's disease in the San Joaquin Valley. Hilgardia 53:1-32.

21. Purcell, A. H., and Hopkins, D. L. 1996. Fastidious xylem-limited bacterial plant pathogens. Annu. Rev. Phytopathol. 34:131-151.

22. Purcell, A. H., Suslow, K. G., and Klein, M. 1994. Transmission via plants of an insect pathogenic bacterium that does not move or multiply in plants. Microb. Ecol. 27:19-26.

23. Raju, B. C., Goheen, A. C., and Frazier, N. W. 1983. Occurrence of Pierce's disease bacteria in plants and vectors in California. Phytopathology 73: 1309-1313.

24. Raju, B. C., Nomé, S. F., Docampo, D. M., Goheen, A. C., Nyland, G., and Lowe, S. K. 1980. Alternative hosts of Pierce's disease of grapevines that occur adjacent to grape growing areas in California. Am. J. Enol. Vitic. 31:144-148.

25. Severin, H. H. P. 1949. Transmission of the virus of Pierce's disease by leafhoppers. Hilgardia 19:190-202.

26. Wells, J. M., Raju, B. C., Hung, H. Y., Weisberg, W. G., Mandelco-Paul, L., and Brenner, D. J. 1987. Xylella fastidiosa new-genus new-species gramnegative xylem-limited fastidious plant bacteria related to Xanthomonas spp. Int. J. Syst. Bacteriol. 37:136-143. 